\title{
Modeling the Sun's open magnetic flux
}

\author{
M. Schüssler ${ }^{1}$ and I. Baumann ${ }^{2}$ \\ 1 Max-Planck-Institut für Sonnensystemforschung, 37191 Katlenburg-Lindau, Germany \\ e-mail: msch@linmpi.mpg.de \\ 2 Royal Observatory of Belgium, Avenue Circulaire 3, 1180 Bruxelles, Belgium
}

Received 21 June 2006 / Accepted 10 August 2006

\begin{abstract}
Context. The heliospheric magnetic field can be extrapolated from the photospheric field distribution using models based upon various approximations. Such models are required for the reconstruction of the open solar magnetic flux prior to the time of direct measurements on the basis of surface flux transport simulations.

Aims. We evaluate the consistency of extrapolation models with direct measurements of the heliospheric magnetic field. Furthermore, we study whether extrapolations on the basis of a surface flux transport model for the photospheric magnetic field reproduce the temporal evolution of the measured near-Earth magnetic field.

Methods. We use the potential field source surface (PFSS) model and the current sheet source surface (CSSS) model to extrapolate the heliospheric field on the basis of the Wilcox Solar Observatory (WSO) synoptic maps of the solar surface field from 1976-2005. The results are compared with the near-Earth measurements of the radial heliospheric field and its independence of latitude found with Ulysses. Furthermore, we determine extrapolations on the basis of photospheric flux distributions computed with a surface flux transport code, using as input sunspot group areas from the SOON database.

Results. The CSSS model based upon WSO data, with a source surface located at $\geq 10 R_{\odot}$ and cusp surface at $1.7 R_{\odot}$, yields the best agreement with the measurements. The flux transport simulations reproduce the observed surface flux together with the open flux if the tilt angle of emerging bipolar magnetic regions is smaller than commonly assumed, but consistent with sunspot observations. Conclusions. The CSSS model with a source surface in the vicinity of the Alfvénic point of the solar wind leads to a good extrapolation of the heliospheric field from solar surface data. A surface flux transport model based upon sunspot data with consistently calibrated tilt angles reproduces the observed evolution of the solar total open flux.
\end{abstract}

Key words. Sun: magnetic fields - Sun: corona

\section{Introduction}

The fraction of the Sun's magnetic surface flux which is not contained in closed loops but reaches out far into the heliosphere is denoted as the Sun's open magnetic flux. Measurements outside the ecliptic plane with the Ulysses space probe covering nearly the full range of heliographic latitudes have shown that the strength of the radial component of the heliospheric field is largely independent of latitude (except for a sudden polarity change at the heliospheric current sheet), so that the solar open flux is almost uniformly distributed on spherical surfaces. This result holds during solar activity minimum (Smith \& Balogh 1995; Balogh et al. 1995) as well as solar maximum (Smith et al. 2003; Smith \& Balogh 2003) periods. It is consistent with the dominance of the heliospheric current for shaping the heliospheric field (e.g., Wolfson 1985) into a split monopole configuration (Banaszkiewicz et al. 1998).

The latitude-independence of the radial heliospheric field component gives the opportunity to infer the total open flux and its time variation on the basis of the interplanetary magnetic field measured near Earth. The results can then be used to test and calibrate models that extrapolate the heliospheric field from its photospheric sources. Such calibration is particularly important for reconstructing the solar open flux in the past with solar surface flux transport simulations based upon sunspot group areas, sunspot numbers, or other proxies. In this way, the simplified reconstruction models of Solanki et al. $(2000,2002)$ can be validated and improved.

The present paper addresses two topics. Firstly, we study the latitude distribution and the time evolution of the radial component of the heliospheric magnetic field arising from extrapolations on the basis of the observed photospheric field. In particular, we consider the potential field source surface model (PFSS, Schatten et al. 1969) and the current sheet source surface model (CSSS, Zhao \& Hoeksema 1995) and study, under which conditions such models can reproduce the latitude-independent field found with Ulysses and the time evolution of the interplanetary field near Earth shown by the OMNI dataset over the last three solar cycles (see also Wang 1993; Wang \& Sheeley 1995, 2002; Wang et al. 2002).

The second (and main) focus of this paper is on the question whether photospheric flux distributions from surface flux transport simulations coupled with extrapolation models can reproduce the heliospheric field measurements over multiple solar cycles. Mackay et al. (2002b) found that flux transport simulations with commonly used prescriptions for the transport processes leads to the open flux being in antiphase with the activity cycle, which is in disagreement with the observations. We show here that this result is probably due to a too steep latitude profile for the tilt angle of bipolar magnetic regions with respect to the East-West direction. This tilt is the key ingredient for the reversal and build-up of opposite-polarity polar fields in the course 
of the solar cycle. We derive the tilt angle profile consistently through comparison of flux transport simulations with photospheric field observations, resulting in a less steep profile than commonly used. With such calibration, the time evolution of the solar open flux throughout the last three solar cycles is well reproduced by the flux transport model, without requiring further adjustments.

This paper is organized as follows. In Sect. 2, we give a brief description of the two extrapolation models used. We present our results in Sect. 3, beginning with the extrapolations from observed photospheric fields in Sect. 3.1, followed by the calibration of the flux transport model and the determination of the open flux on the basis of simulated photospheric flux distributions in Sect. 3.2. We discuss the results and give our conclusions in Sect. 4.

\section{Extrapolation methods}

\subsection{Potential field source surface (PFSS) model}

In this widely used model, originally proposed by Schatten et al. (1969) and Altschuler \& Newkirk (1969), the coronal magnetic field is assumed to be potential and can thus be obtained by solving the Laplace equation in a spherical shell bounded by the photosphere and the source surface (usually placed at $R_{\mathrm{ss}}=2.5 R_{\odot}$ ), where the magnetic field is assumed to be purely radial, being dragged out by the solar wind in the region beyond the source surface. The solution for the radial magnetic field component on the source surface is expressed in the form

$B_{\mathrm{r}}\left(R_{\mathrm{ss}}, \lambda, \phi\right)=\sum_{l=0}^{\infty} \sum_{m=-l}^{m=l} a_{l m} c_{l}\left(R_{\mathrm{ss}}\right) Y_{l m}(\lambda, \phi)$,

where $\lambda$ and $\phi$ are the heliographic latitude and longitude, respectively, and $Y_{l m}(\lambda, \phi)$ are the spherical surface harmonics. The quantities

$a_{l m}=\int B_{\mathrm{r}}\left(R_{\odot}, \lambda, \phi\right) Y_{l m}^{*}(\lambda, \phi) \mathrm{d} \Omega$

are the complex harmonic coefficients of the surface magnetic field, and

$c_{l}\left(R_{\mathrm{ss}}\right)=\left(\frac{R_{\odot}}{R_{\mathrm{ss}}}\right)^{l+2}\left[\frac{l+1+l\left(r / R_{\mathrm{ss}}\right)^{2 l+1}}{l+1+l\left(R_{\odot} / R_{\mathrm{ss}}\right)^{2 l+1}}\right]$

are the radial damping coefficients (e.g., Wang \& Sheeley 1992).

\subsection{Current sheet source surface (CSSS) model}

In order to improve the PFSS model, particularly concerning the topology of the field near coronal streamers, Schatten (1972) suggested an extrapolation model that allows for current sheets. In a first step, a potential field solution is derived for the whole external region. Then a spherical "cusp surface" is defined (located at $R_{\text {cusp }}<2 R_{\odot}$ ), beyond which no closed field lines are assumed to exist. In order to calculate such a solution, inward pointing field vectors on the cusp surface are temporarily reversed and, based upon this boundary condition, a new potential field solution is calculated outside the cusp surface. Thereafter, the condition $\nabla \cdot \boldsymbol{B}=0$ is restored by reinstating the original orientation of the field vectors. This creates discontinuities (corresponding to the locations of current sheets) in the field distribution outward of the cusp surface. The procedure is justified by the fact that the Maxwell stress tensor is independent of the sign of the magnetic field.

Zhao \& Hoeksema (1994) replaced the potential field in the region inward of the cusp surface by a magnetostatic model of the corona. Later, these authors extended their model by reintroducing a source surface with purely radial magnetic field, thus creating the current sheet source surface (CSSS) model (Zhao \& Hoeksema 1995). Here we consider the latter model in addition to the PFSS model.

We should note that, owing to the restoration of the original field direction on the cusp surface, the sign of the field vectors beyond the cusp surface and the location of the current sheets has to be determined by field line tracing. Since here we are only interested in the unsigned open flux and its latitude distribution, we have not carried out this procedure and thus always consider only the absolute value of the extrapolated radial field strength.

\subsection{Open flux and field strength near Earth}

The total unsigned open flux provided by the extrapolations is calculated by integrating the unsigned radial magnetic field component over the source surface,

$\Phi_{\text {open }}(t)=R_{\mathrm{ss}}^{2} \int_{0}^{\pi} \int_{0}^{2 \pi}\left|B_{\mathrm{r}}\left(R_{\mathrm{ss}}, \lambda, \phi, t\right)\right| \cos \lambda \mathrm{d} \lambda \mathrm{d} \phi$.

In the case of the CSSS model, the open flux is already determined at the cusp surface, so that one could as well carry out the integration over that surface. We also define the mean flux density at the radial distance of the Earth, $r_{\mathrm{E}}=1 \mathrm{AU}$, by

$\left\langle B_{\mathrm{E}}\right\rangle(t)=\frac{\Phi_{\mathrm{open}}(t)}{4 \pi r_{\mathrm{E}}^{2}}$.

If the model is consistent with the Ulysses result of a latitudeindependent field, $\left\langle B_{\mathrm{E}}\right\rangle(t)$ is equal to the longitudinally averaged field near Earth (at $r=r_{\mathrm{E}}$ and $\lambda=0$, ignoring the slight variation due to the $b$ angle, i.e., the angle of about 7 degrees between the equatorial plane of the Sun and the ecliptic),

$B_{\mathrm{E}}(t)=\frac{1}{2 \pi}\left(\frac{R_{\mathrm{ss}}}{r_{E}}\right)^{2} \int_{0}^{2 \pi}\left|B_{\mathrm{r}}\left(R_{\mathrm{ss}}, 0, \phi, t\right)\right| \mathrm{d} \phi$.

\section{Results}

In order to investigate the open flux and the latitude dependence of the magnetic field beyond the source surface resulting from the extrapolation models, the photospheric source field has to be provided as a boundary condition. Here we consider observational data in the form of the synoptic maps from the Wilcox Solar Observatory for the time period 1976-2005 as well as results from flux transport simulations based upon the sunspot group areas from the USAF/NOAA Solar Optical Observing Network (SOON) dataset.

For the location of the source surface, we have used the "standard" value of $2.5 R_{\odot}$ in the case of the PFSS model, for which value the positions of the coronal holes and the polarity pattern of the interplanetary magnetic field apparently are well reproduced (Hoeksema 1984). Assuming a uniform latitudinal distribution of the open flux (which the PFSS model in fact does not yield, as we shall see below), this choice gives a reasonable agreement with the measurements of the near-Earth field (Wang $\&$ Sheeley 1995). For the CSSS model, the total open flux is already determined at the location of the cusp surface, for which 
we chose the value $R_{\text {cusp }}=1.7 R_{\odot}$. The resulting total open flux is then similar to that given by the PFSS model. The location of the source surface in the CSSS model then "freezes" the latitude distribution of the open flux further outward. We shall see that chosing a value of $2.5 R_{\odot}$ (Zhao \& Hoeksema 1995) leads to a latitude distribution that is inconsistent with the Ulysses result, similar to the case of the PFSS model. On the other hand, the physical justification for introducing the source surface is the control of the radially streaming solar wind over the magnetic field beyond the Alfvén radius, $r_{\mathrm{A}}$, where the kinetic energy density of the wind surpasses the magnetic energy density. The best existing estimates put $r_{\mathrm{A}}$ in the range $10 \ldots 30 R_{\odot}$ (Marsch \& Richter 1984). We have therefore considered also larger source surface radii in the case of the CSSS model.

\subsection{Extrapolation based on WSO synoptic maps}

The spherical harmonic components of the synoptic photospheric field maps from Wilcox Solar Observatory (WSO) are available online ${ }^{1}$ up to order $l=9$ for the Carrington rotations 1641-2035, covering the time period between 1976 and 2005. We have used the coefficients obtained with the socalled radial boundary condition and corrected the data for the FeI $5250 \AA$ line saturation by applying the latitude-dependent factor $\delta^{-1}=4.5-2.5 \sin ^{2} \lambda$ according to Ulrich (1992) and Ulrich et al. (2002).

\section{Latitudinal variation}

Latitudinal profiles of the (unsigned) radial field at the source surface resulting from extrapolations with the PFSS and the CSSS models are shown in Fig. 1. For each model we have chosen three Carrington rotations during solar maximum periods (CR 1688, 1819 and 1961) and solar minimum periods (CR 1643, 1781 and 1909), respectively.

During solar minima, the source surface field obtained from both models with $R_{\mathrm{ss}}=2.5 R_{\odot}$ (top and middle row of Fig. 1) shows a very strong decrease between poles and equator. During solar maxima, the latitudinal variations are less systematic but still significant. These results are in clear contradiction to the Ulysses measurements of a practically latitude-independent field. However, by placing the source surface out to $10 R_{\odot}$ and beyond in the CSSS model, we obtain an almost constant field, during maximum as well as minimum periods (bottom row of Fig. 1). The near-equator current sheet in the CSSS model indicated by the finite value of $B_{\mathrm{r}}$ for all latitudes permits the development of a "split monopole" configuration (Banaszkiewicz et al. 1998) if the source surface is located sufficiently far from the solar surface. Such a configuration, which is required to reproduce the Ulysses result, cannot be achieved by a PFSS extrapolation model.

\section{Open flux and near-Earth field}

Figure 2 shows the time evolution of the mean unsigned radial flux density at $1 \mathrm{AU}$ (proportional to the total open flux, cf. Lockwood et al. 2004), $\left\langle B_{\mathrm{E}}\right\rangle$, as defined in Eq. (5) and the near-Earth field, $B_{\mathrm{E}}$. These quantities are given as 3-month averages in comparison with the measured radial component of the near-Earth interplanetary magnetic field (dotted lines) from GSFC/SPDF OMNI ${ }^{2}$ data.

\footnotetext{
1 http://soi.stanford. edu/ wso/Harmonic . rad

${ }^{2}$ http://omniweb.gsfc.nasa.gov
}

The mean radial field $\left\langle B_{\mathrm{E}}\right\rangle$ matches the measurements reasonably well for both models, indicating that they reproduce the temporal evolution of the total open magnetic flux. However, the predicted field strength near the ecliptic, $B_{\mathrm{E}}$, is badly reproduced for the PFSS model as well as for the CSSS model if the source surface is placed at the "standard" distance of $R_{\mathrm{ss}}=2.5 R_{\odot}$. While we cannot change this value in the PFSS model because then the total open flux would take wrong values, we have that freedom in the CSSS model since here the open flux is already fixed by the choice of the cusp surface at $1.7 R_{\odot}$. Taking $R_{\mathrm{ss}}=10 R_{\odot}$, which is supported by the physical arguments discussed at the beginning of Sect. 3 , leads to consistency of the total open flux as well as the near-Earth field with the observed data. Consequently, while the PFSS model also yields the correct result for the total open flux, only the CSSS model reproduces the latitude-independent radial field as observed with Ulysses.

\subsection{Extrapolation based on flux transport simulations}

Flux transport models describe the evolution of magnetic flux on the solar surface, which emerges in form of bipolar magnetic regions. Such models are used to follow the temporal evolution of the radial magnetic field under the influence of turbulent diffusion (caused by supergranulation) and of horizontal flows due to differential rotation and meridional circulation (e.g., Leighton 1964; DeVore et al. 1984; Wang et al. 1989; van Ballegooijen et al. 1998; Schrijver 2001; Mackay et al. 2002b; Baumann et al. 2004, 2006).

\section{Flux transport model}

We simulate the evolution of the radial surface field with the flux transport code and the representation of emerging bipoles as described in Baumann et al. (2004), including the decay term representing the effect of radial diffusion with $\eta=100 \mathrm{~km} \mathrm{~s}^{-2}$ (Baumann et al. 2006). We use a value of $600 \mathrm{~km}^{2} \mathrm{~s}^{-1}$ for the surface diffusivity and the differential rotation profile derived by Snodgrass (1983). For the meridional flow velocity we use a semi-empirical latitudinal profile adapted to helioseismic measurements (Gizon \& Duvall 2004):

$v(\lambda)=1.6 \sin (2 \lambda) \exp [\pi(1-|\lambda| / 90)]$

in units of $\mathrm{m} \mathrm{s}^{-1}$. This profile and the helioseismic data are shown in Fig. 3, together with the profiles adopted in other studies.

We have derived the flux emergence data required as input for the model from the sunspot group record provided by the USAF/NOOA Solar Optical Observing Network (SOON) ${ }^{3}$. Sunspot areas (as well as sunspot numbers, see Solanki et al. 2000, 2002) represent a good proxy for flux emergence because sunspots typically appear in the early phases of active regions and most of them have a lifetime of only a few days. We have used the SOON data (courtesy of D. Hathaway, NASA/MSFC) in order to derive a sequence of emerging bipolar magnetic regions (BMRs) as input for the flux transport simulation according to Baumann et al. (2004). The basic assumptions are: (i) every observed sunspot group corresponds to one BMR, and (ii) the magnetic flux content of a BMR is proportional to its area (Schrijver \& Harvey 1994). Since a sunspot group typically appears more than once in the data, we consider a group only at the day of its maximum area.

\footnotetext{
${ }^{3}$ http://solarscience.msfc.nasa.gov/greenwch. shtml
} 

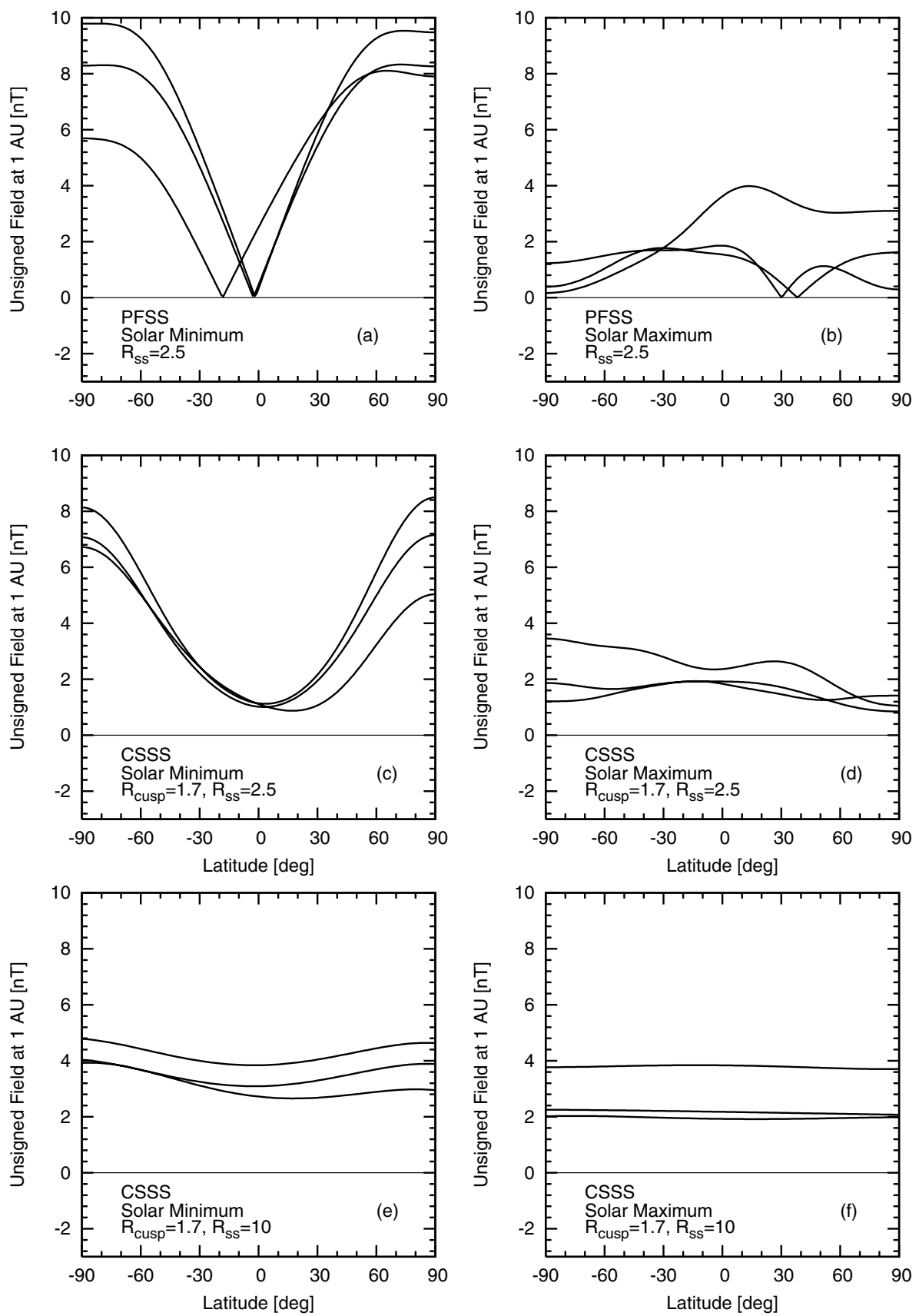

Fig. 1. Latitude dependence of the longitudinally averaged source surface field, $\left|B_{\mathrm{r}}\left(R_{\mathrm{ss}}, \lambda\right)\right|$, during times of three solar activity minima (Carrington rotations 1643, 1781, 1909; left panels) and three maxima (Carrington rotations 1688, 1819, 1961; right panels), respectively, obtained on the basis of the WSO photospheric field maps. Cases considered are the PFSS model with $\left.R_{\mathrm{ss}}=2.5 R_{\odot}(\mathbf{a}), \mathbf{b}\right)$ ), the CSSS model with $R_{\text {cusp }}=1.7 R_{\odot}$ and $\left.R_{\mathrm{ss}}=2.5 R_{\odot}(\mathbf{c}), \mathrm{d}\right)$ ), as well as the CSSS model with $R_{\text {cusp }}=1.7 R_{\odot}$ and $R_{\mathrm{ss}}=10 R_{\odot}(\mathbf{e})$,f)). The Ulysses result of a latitude-independent field is only reproduced by a CSSS model with a source surface located in the region of the Alfvénic point, $r \geq 10 R_{\odot}$.

For the area of a BMR, $A_{\mathrm{BMR}}$, we write $A_{\mathrm{BMR}}=A_{\mathrm{s}}+A_{\mathrm{f}}$, where $A_{\mathrm{s}}$ is the sunspot group area and $A_{\mathrm{f}}$ is the corresponding facular area of the bipolar region given by the relation

$A_{\mathrm{f}}=414+21 A_{\mathrm{s}}-0.0036 A_{\mathrm{s}}^{2}$,

in millionths of a solar hemisphere. This relationship has been derived observationally by Chapman et al. $(1997,2001)$.

The sunspot group data contain only information about the visible hemisphere of the Sun. Consequently, the observations probably miss up to $50 \%$ of the small sunspots. For larger sunspot groups, this fraction is smaller because such groups emerging on the far side of the Sun may live sufficiently long to rotate to the visible hemisphere and thus become observable. The estimation of the correct number of missing spot groups in the observation is a difficult task, since one has to take into account visibility problems near the solar limb, different decay stages of the spot groups, and the recurrence of sunspot groups (see, e.g., Kopecký et al. 1985). For our aims, we restrict ourselves to a rough estimation of the missing spot groups by assuming a sunspot group observed at longitude $\phi_{0}$ to emerge as well at longitude $\phi_{0}+180^{\circ}$, with equal size and at the same latitude. This procedure will somewhat overestimate the contribution of very large sunspot groups. On the other hand, recurrent flux emergence (nests of activity) within the same area could 

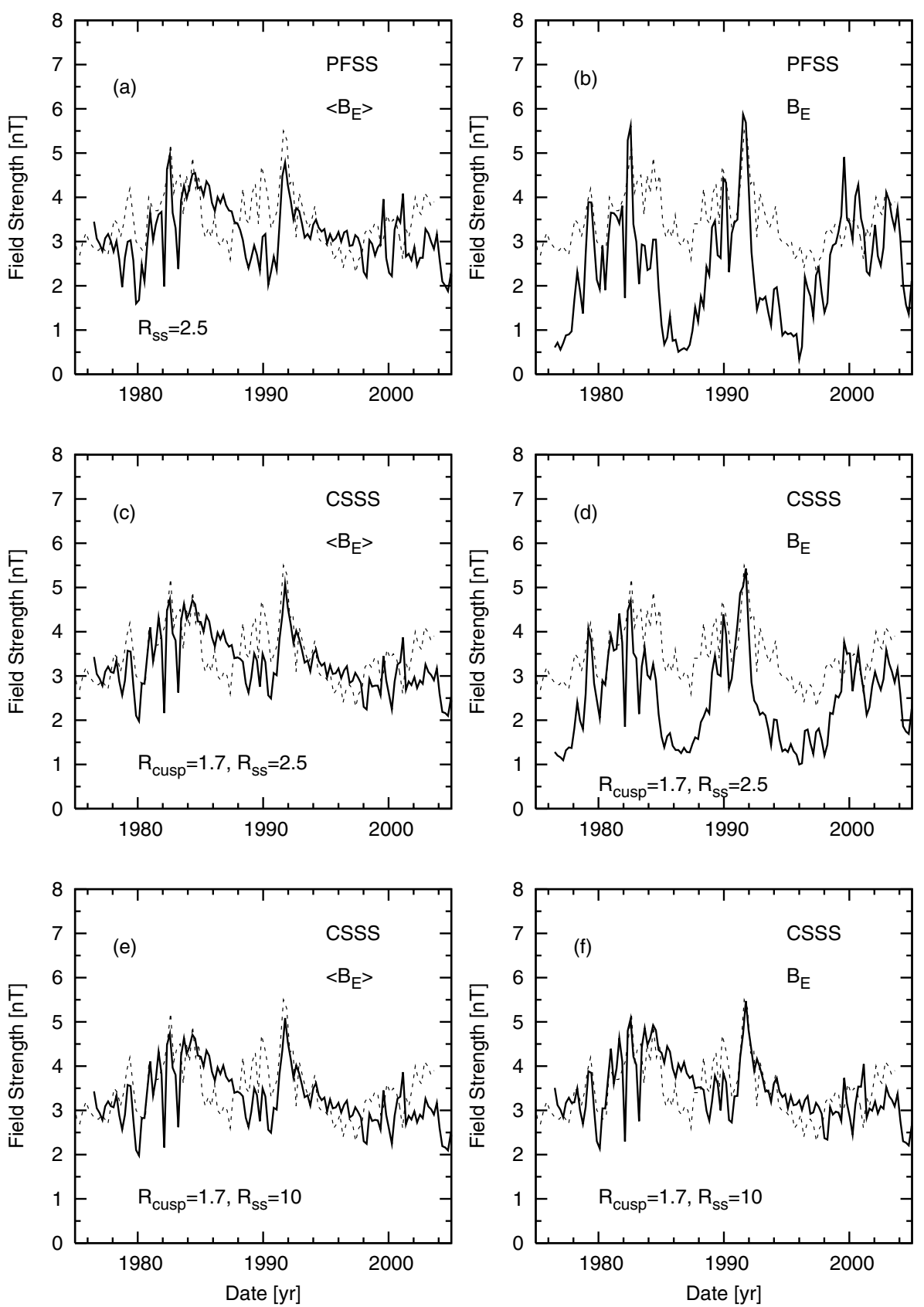

Fig. 2. Mean radial flux density at $1 \mathrm{AU}\left(\left\langle B_{\mathrm{E}}\right\rangle\right.$, see Eq. (5), left panels) and radial field near Earth ( $B_{\mathrm{E}}$, see Eq. (6), right panels) for the same extrapolations as in Fig. 1 (solid lines) in comparison with the measured radial IMF component (OMNI data, dotted lines). The extrapolations are based on the WSO synoptic maps of the photospheric magnetic field. All data are 3-month averages. The results for $\left\langle B_{\mathrm{E}}\right\rangle$ (proportional to the total open flux) fit the data reasonably well for PFSS as well as CSSS extrapolations. However, only in the case of CSSS with $R_{\mathrm{sS}}=10 R_{\odot}$ (panel f)) is the extrapolated near-ecliptic field consistent with the actual data.

possibly be missed by our procedure, which takes into account only the maximum area of a sunspot group throughout its whole evolution.

Since the SOON data give no information about magnetic polarity, we infer the polarities of the BMRs through Hale's rules (Hale \& Nicholson 1925) applied to each cycle.

\section{Tilt angle}

The angular deviation from the East-West direction of the line joining the centroids of the two polarities of a BMR (leading and following parts of the sunspot groups), the tilt angle $\gamma$, is crucial for the evolution of the polar fields and the solar axial dipole (e.g., Mackay et al. 2002a). Since no tilt angle information is given in the SOON data, we assume a latitude dependence of the form $\gamma=f \lambda$. The factor of proportionality, $f$, is left as a parameter to be fixed by comparison with the observed surface fluxes (see below). A number of earlier studies with flux transport models have used $f=0.5$ as proposed by Wang \& Sheeley (1989) on the basis of magnetograph data, but our results below suggest a considerably smaller value.

In order to fix the value of $f$, we calculate the longitude-averaged photospheric radial surface field, take its modulus and integrate over latitude to obtain $B_{\mathrm{L}}=$ $(4 \pi)^{-1} \int\left|\int B_{r}(\lambda, \phi) \mathrm{d} \phi\right| \cos \lambda \mathrm{d} \lambda$. This quantity is very sensitive to the tilt angle of the BMRs. In fact, for the small tilt angles 


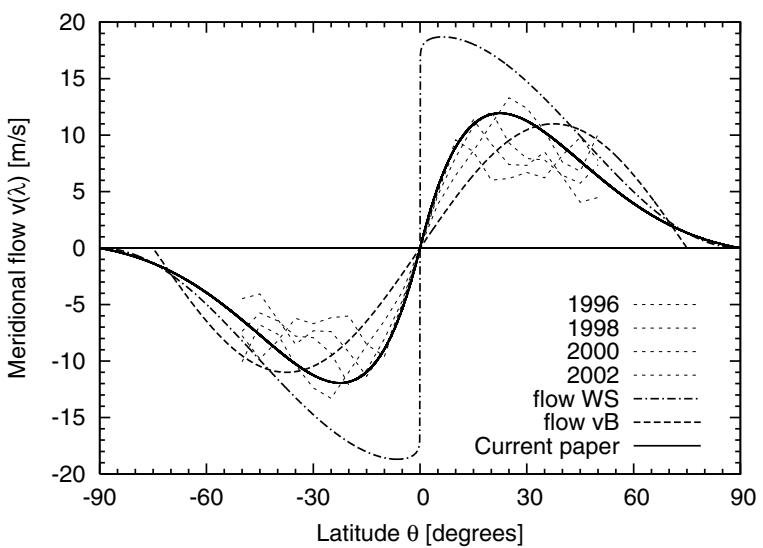

Fig. 3. Latitude profiles of the meridional flow velocity. The profile used for the simulations presented here (solid line) has been roughly adapted to helioseismological measurements (dashed lines with year labels, cf. Gizon \& Duvall 2004). Also shown are the profiles adopted in other studies with flux transport models. flow WS: Wang \& Sheeley (2003a) and Wang et al. (2005); flow vB: van Ballegooijen et al. (1998) and Baumann et al. (2004).

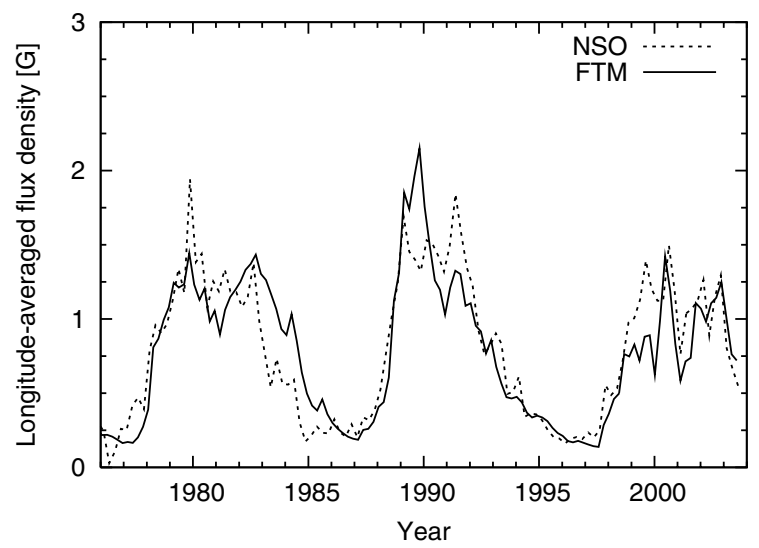

Fig. 4. Longitude-averaged photospheric magnetic field, unsigned and integrated over latitude, as a function of time (three-months averages). Shown are the result from our flux transport simulations (FTM, full line) with sources taken from the SOON sunspot area database together with the observed data (dotted line) based upon the NSO/Kitt peak synoptic magnetograms (courtesy of D. Hathaway, NASA/MSFC). The conversion factor between bipolar magnetic region area and input magnetic flux for the flux transport simulations has been chosen such that the two curves show a reasonable agreement. The calibrated curves for different values of the proportionality factor, $f$, in the latitudinal tilt angle profile, $\gamma=f \lambda$, are nearly identical for $f \leq 0.5$. Shown here is the curve for $f=0.15$.

relevant here, we have $B_{\mathrm{L}} \propto f$. Therefore, for a given value of $f$ we can calibrate the (still unspecified) conversion factor between BMR area, $A_{\mathrm{BMR}}$, and its magnetic flux by comparing the simulated with the observed time evolution of $B_{\mathrm{L}}$. This is shown in Fig. 4 for the period 1976-2004 with observational data derived from NSO Kitt Peak synoptic magnetgrams. The calibrated curves for different values of $f$ are almost identical, but the conversion factors are inversely proportional to $f$. The actual curve shown in Fig. 4 is for $f=0.15$.

Having determined the linear relationship between the areaflux conversion factor and $f$, we use the mean unsigned surface flux density, $B_{\mathrm{s}}=(4 \pi)^{-1} \iint\left|B_{r}(\lambda, \phi)\right| \cos \lambda \mathrm{d} \lambda \mathrm{d} \phi$ to fix the correct value of $f$. This is shown in Fig. 5, which gives three-month averages of the simulation results for $f=0.15$ (solid curve) and

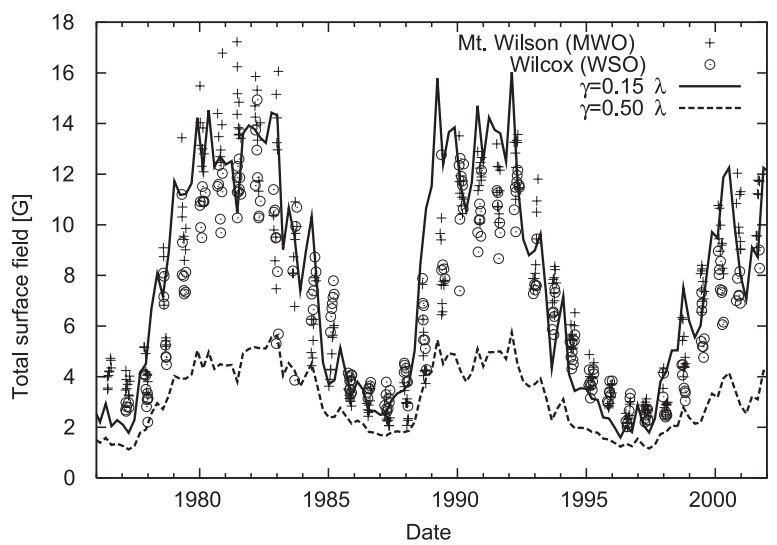

Fig. 5. Averaged unsigned photospheric field as a function of time. The symbols represent data from Mt. Wilson Observatory and Wilcox Solar Observatory, respectively (cf. Arge et al. 2002). The curves give three-months averaged results from flux transport simulations for two values of the proportionality factor, $f$, in the latitudinal tilt angle profile, calibrated by requiring consistency with the longitudinally averaged flux density (see Fig. 4). While the curve for $f=0.5$ (dashed line) shows a much too small average surface field, the result for $f=0.15$ (solid line) is consistent with the data.

for $f=0.5$ (dashed curve). The symbols show the corresponding observations from the Mount Wilson Observatory and Wilcox Solar Observatory magnetograph data (cf. Arge et al. 2002). It is obvious that the case $f=0.5$ shows a much too small surface flux while the case $f=0.15$ fits the data well, without requiring any further calibration. Therefore, requiring the consistency of both, $B_{\mathrm{L}}$ and $B_{\mathrm{s}}$, with the observational data uniquely fixes the tilt angle law to $\gamma=0.15 \lambda$. This value is consistent with the analysis of sunspot data (Howard 1991; Sivaraman et al. 1999) as well as with results from numerical simulations of rising flux tubes (e.g., Caligari et al. 1995).

Figure 6 gives time-latitude diagrams of the azimuthally averaged radial surface field from the flux-transport model (with $f=0.15$, calibrated as described above) and from observational results. Both diagrams show a reasonable agreement, particularly concerning the polar field reversals shortly after the activity maxima and the poleward surges of following-polarity magnetic flux leading to these reversals. Figure 7 shows the temporal evolution of the field strength on both polar caps (poleward of \pm 70 deg latitude), which is consistent with the observational results presented by Dikpati et al. (2004).

\section{Latitudinal variation, open flux, and near-Earth field}

The latitudinal profiles of the unsigned radial field at the source surface based on extrapolations of the photospheric fields from the flux transport simulations show the same characteristics as those for the WSO data given in Fig. 1: only the CSSS model with a source surface located at $10 R_{\odot}$ (or beyond) reproduces a latitude-independent radial field in the heliosphere as observed with the Ulysses probe. This is not surprising since the latitude dependence of the heliospheric field is largely determined by the extrapolation model and not by the detailed distribution of the photospheric sources.

Figure 8 gives the time evolution between 1976 and 2004 of $\left\langle B_{\mathrm{E}}\right\rangle$, the mean unsigned radial field at $1 \mathrm{AU}$, which is proportional to the total open flux (Lockwood et al. 2004). Shown are the CSSS extrapolations (with $R_{\mathrm{ss}}=2.5 R_{\odot}$ and $R_{\text {cusp }}=$ $10 R_{\odot}$, solid lines) based upon the flux transport simulations for 

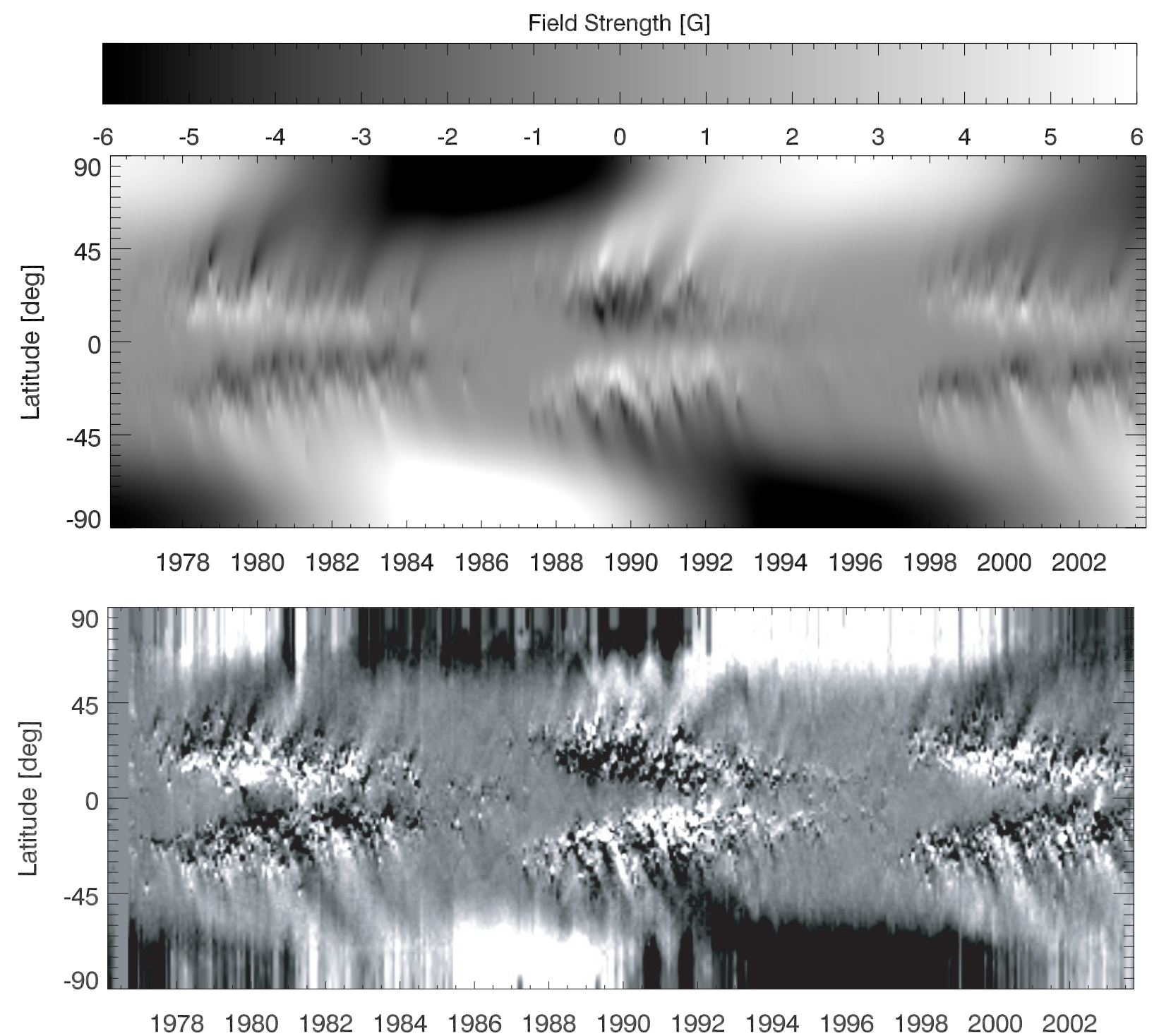

Fig. 6. Simulated and observed time-latitude plots (butterfly diagrams) of the longitudinally averaged radial magnetic field at the solar surface. Upper panel: result of the flux transport simulation (running time averages of over 27 days) based upon SOON sunspot data with a value of $f=0.15$ for the tilt angle slope. Lower panel: evolution of the observed field from NSO Kitt Peak synoptic maps (courtesy D. Hathaway). Simulation and observation are in qualitative agreement, particularly concerning the poleward surges of following-polarity magnetic flux leading to the reversals of the polar fields.

$f=0.15$ (upper panel) and $f=0.5$ (lower panel), respectively. Dashed lines give the measurements (OMNI data). It is clear that the simulation with $f=0.5$ fails to reproduce the measurements, while for $f=0.15$ the agreement is quite satisfactory, apart from somewhat too low values during the rising phases of the cycles. Note that no further calibration or adjustment has been made in order to achieve this agreement.

\section{Discussion and conclusions}

We have addressed the latitude dependence of the heliospheric field and the total open solar flux as resulting from extrapolation models. The photospheric field distribution has been taken either from observations (WSO data) or from flux transport simulations with flux emergence determined from the SOON sunspot group database.

We have confirmed that the widely used potential field source surface model (PFSS) cannot reproduce the latitudeindependent radial field in the heliosphere as found by
Ulysses measurements. Other researchers already have noted this fact (e.g., Wang 1993; Wang \& Sheeley 1995, 2002) and suggested that the heliospheric current could be responsible for transforming the field configuration into the observed "split monopole" configuration. We have shown here, in the framework of the current sheet source surface (CSSS) model of Zhao \& Hoeksema (1995), that the effect of the current sheet has to be combined with a source surface located in the region of the Alfvénic surface beyond $10 R_{\odot}$ in order to obtain a latitudeindependent radial field. Such a location of the source surface makes physical sense because the field can be expected to be drawn out radially only when the kinetic energy density of the solar wind dominates over the magnetic energy density.

With a latitude-independent radial field, the time series of field measurements near Earth also represents the evolution of the open flux. It turns out that the total open flux determined by a PFSS extrapolation on the basis of the WSO magnetograms of the photospheric field can reproduce the measurements when it is assumed that the open flux becomes distributed uniformly 


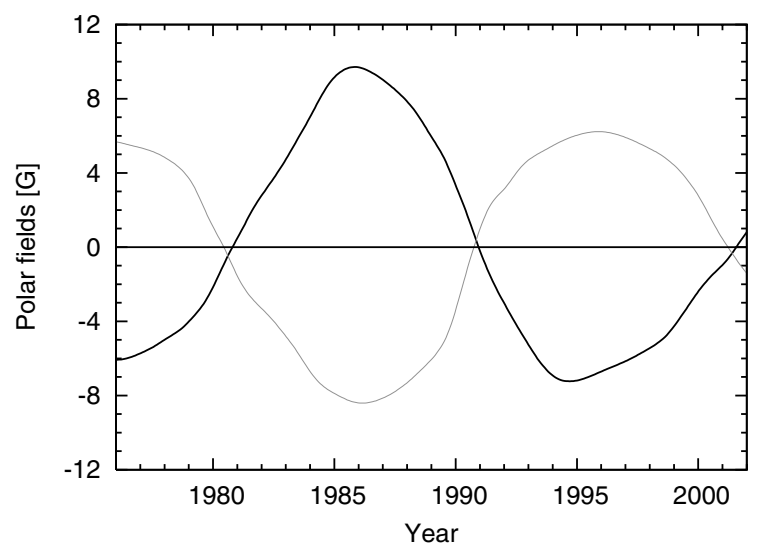

Fig. 7. Evolution of polar fields (average field strength poleward of \pm 70 deg latitude) in the flux transport simulation. South (North) polar fields are indicated by the thick (thin) line. The field amplitudes and reversal times are consistent with the observational results presented by Dikpati et al. (2004, cf. their Fig. 3). Owing to the calibration of the flux transport model (see Fig. 4), the curves for different values of the proportionality factor, $f$, in the latitudinal tilt angle profile, $\gamma=f \lambda$, are nearly identical as long as $f \leq 0.5$. Shown here is the case $f=0.15$.

in latitude beyond the source surface (Fig. 2a, see also Wang \& Sheeley 1995, 2002). Consequently, the choice of the source surface radius $R_{\mathrm{ss}}=2.5 R_{\odot}$ in the PFSS seems seems to capture the right amount of amount of open flux. We have shown here that an extrapolation fully consistent with both, the time evolution of the near-Earth radial field (also representing the open flux) as well as the latitude independence of the heliospheric radial field, can be obtained with the CSSS model incorporating the heliospheric current sheet and a source surface in the vicinity of the Alfvénic surface beyond $10 R_{\odot}$.

The second topic of this paper is the question under which conditions flux transport simulations can reproduce the observed characteristics of the evolution of the total open flux during the solar cycle. Mackay et al. (2002b) have noted that with standard assumptions concerning diffusivity, meridional flow, tilt angle, and latitude distribution of emerging flux (butterfly diagram) the open flux is in antiphase with the activity cycle, i.e., it peaks during solar activity minima, in contradiction to the measurements (see also Fig. 2 in Wang et al. 2002). This result indicates a too strong weighting of the axial dipole field (polar field) during solar minima with respect to the contribution of the low-latitude fields (active regions and their remnants, equatorial dipole) in the standard flux transport simulations. In fact, Wang \& Sheeley (2003a) and Schrijver \& DeRosa (2003) have shown on the basis of potential-field models that the open flux around solar activity maximum is strongly affected by low-latitude active region emergence. Mackay \& van Ballegooijen (2006) have confirmed and substantiated this result with non-potential simulations of interacting bipoles.

Because the extrapolation models based on actual observations of the photospheric field successfully reproduce the observed time evolution of the total open flux, including its phase relation with activity cycle, the problem probably does not arise from the extrapolation models. Wang et al. (2002) have suggested a combination of increased source strength (amplifying the low-latitude contribution) and a strong meridional flow with a particular latitude profile, which reduces the polar fields by suppressing the diffusion of magnetic flux across the equator. They find that a PFSS extrapolation with this "revised model" reproduces the observed evolution of the open flux in solar
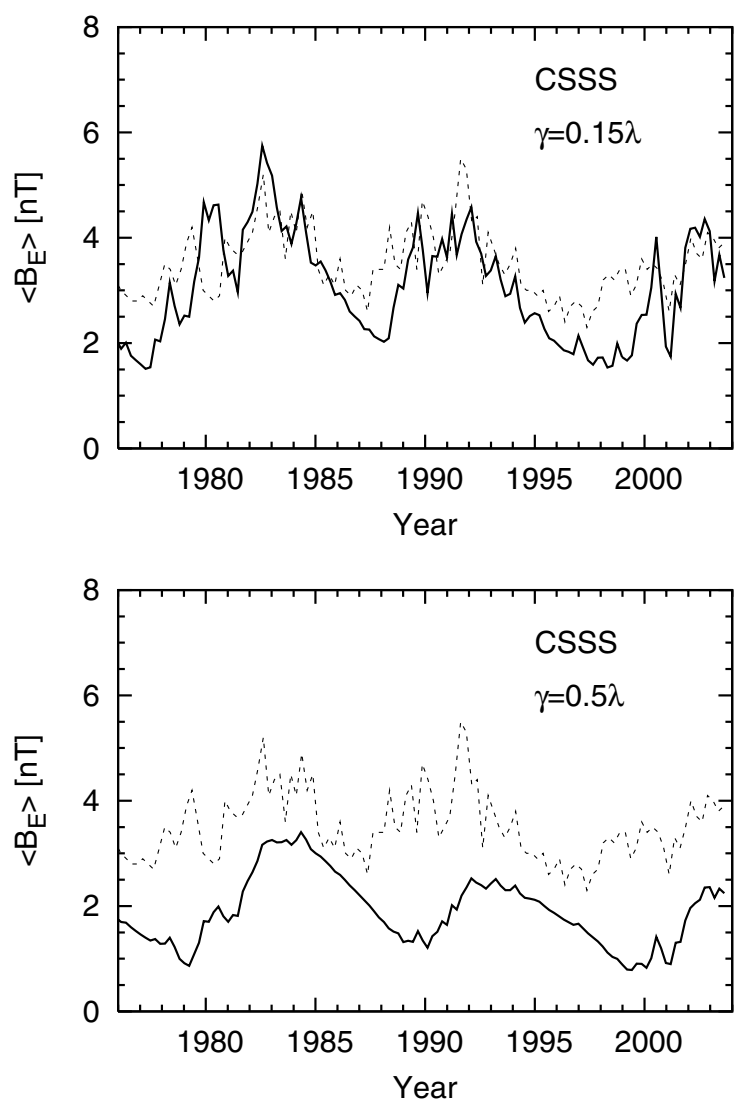

Fig. 8. Temporal evolution of the mean unsigned radial field at $1 \mathrm{AU}$ : comparison between measurements (dashed lines, OMNI data) and CSSS extrapolations (solid lines, $R_{\text {cusp }}=1.7 R_{\odot}, R_{\mathrm{ss}}=10 R_{\odot}$ ) based on flux transport simulations with input from the SOON sunspot group areas. The upper panel shows the case $f=0.15$ (tilt angle profile as consistently determined by comparison with observed surface fluxes, see Figs. 4 and 5). The lower panel shows the case $f=0.5$. In this case, the overall amplitude of the open flux is too low. In addition, there is a phase shift between the curves, although they are not in antiphase (see, e.g., Mackay et al. 2002b) owing to the effect of the decay term in the flux transport equation (Baumann et al. 2006). Clearly, the simulation with $f=0.15$ yields a reasonable representation of the measurements.

cycle 21 . However, the assumed profile of the meridional flow is at variance with the results from helioseismology (see curve "flow WS" in Fig. 3).

We have shown that the problem can be solved without modifying the meridional flow profile. We have abandoned the commonly used value $f=0.5$ of the proportionality factor in the latitude dependence of the tilt angle, $\gamma=f \lambda$ (Wang $\&$ Sheeley 1989). Instead, we have determined a consistent value of $f$ by requiring that the longitudinally averaged photospheric magnetic flux $\left(B_{\mathrm{L}}\right)$ and the total surface flux $\left(B_{\mathrm{S}}\right)$ from the simulation simultaneously agree with the observational data. Simulations of the photospheric flux distributions carried out with the value of $f=0.15$ obtained in this way lead to a reasonably good reproduction of the measured near-Earth radial field (see Fig. 8, upper panel) when extrapolated with a CSSS model with a cusp surface at $1.7 R_{\odot}$ (determining the total amount of open flux) and a source surface at $\geq 10 R_{\odot}$, providing a latitude-independent radial field in the heliosphere.

The flatter profile of the tilt angle law suggested by our simulations is consistent with results from sunspot groups (Howard 1991; Sivaraman et al. 1999) as well as with tilt angles from numerical simulations of rising magnetic flux tubes 
(e.g., Caligari et al. 1995). In fact, sunspot groups are very well suited to observationally determine the relevant tilt angles because they represent active regions in their early phases during and after emergence. Later, the configuration of active regions becomes distorted through flux transport on the surface and does no longer necessarily reflect the tilt angle of the emerging flux tube. This probably explains why the sunspot results (e.g., Howard 1991) and our indirect determination of the tilt angle profile differ from the results of Wang \& Sheeley (1989).

Our successful reproduction of the observed variation of the total open flux with realistic assumptions about the flux transport processes indicates that the evolution of the solar open flux is largely determined by the instantaneous photospheric sources. Reconnection processes in the corona (e.g., Fisk 2005) could possibly modify the distribution of the open flux. Such "interchange reconnection" has recently been demonstrated by Mackay \& van Ballegooijen (2006) through simulations of interacting, non-potential bipoles. However, such processes do neither affect the total amount of open flux nor do they change the "split-monopole" configuration at larger distances from the Sun, resulting from the heliospheric current sheet and the dominating, radially streaming solar wind (for a comprehensive and illustrative picture of the changing topology of the coronal field in the course of the solar cycle see Wang \& Sheeley 2003b).

Our model certainly leaves much room for improvement. For instance, the treatment of flux emergence on the invisible hemisphere is rather crude and could be improved (e.g., Kopecký et al. 1985). In the future, far-side helioseismic information can help to cover the full solar surface (e.g., Schrijver \& DeRosa 2003). Our treatment probably somewhat overestimate the contribution of very large sunspot groups, which may have resulted in requiring a slightly flatter tilt angle profile, i.e., somewhat smaller value of $f$, than actually present. This would mainly affect the contribution of BMRs at higher latitudes during the rising phase of the solar activity cycle and could possibly explain the systematically too low values of the simulated open flux during these phases. Furthermore, by the nature of the sunspot group data, complexes of activity with recurrent flux emergence and multiple bipoles may often appear in our source model only as a single large bipole. If the individual bipoles of such a complex show a large scatter of tilt angles, this would also reduce the effective tilt angle associated with the complex of activity. It would certainly be preferable to determine the tilt angles directly from sunspot observations, but such data covering extended periods of time are not available at present. Another unclear point is the temporal variation of the meridional flow and a possible dependence on the activity cycle (e.g., Gizon 2004; Gizon \& Birch 2005), which could also affect the long-term evolution of the open flux (Wang et al. 2002). At present, however, the degree and character of such a variation is still rather uncertain.

We have exclusively used photospheric observational data to calibrate our flux transport model based upon sunspot area data and obtained a good representation of the observed open flux via a CSSS extrapolation. This opens the possibility to extend the model backward in time: first, by using the combined $\mathrm{RGO} / \mathrm{SOON}$ sunspot data reaching back to 1874 and comparing with the open flux reconstructed from the geomagnetic $a a$ index since 1868 by Lockwood et al. (1999); secondly, by using the sunspot number as a proxy for flux emergence and thus extend the model back to the year 1700. These results will be presented in a subsequent paper.
Acknowledgements. We are grateful to Y.-M. Wang and N. R. Sheely for helpful dicussions, to L. Gizon for kindly providing the helioseismic meridional flow data, and to C. N. Arge for making available the corrected data for the total photospheric surface flux. D. Schmitt provided helpful comments on a draft version of the manuscript.

\section{References}

Altschuler, M. D., \& Newkirk, G. 1969, Sol. Phys., 9, 131

Arge, C. N., Hildner, E., Pizzo, V. J., \& Harvey, J. W. 2002, J. Geophys. Res. (Space Physics), 107

Balogh, A., Smith, E. J., Tsurutani, B. T., et al. 1995, Science, 268, 1007 Banaszkiewicz, M., Axford, W. I., \& McKenzie, J. F. 1998, A\&A, 337, 940 Baumann, I., Schmitt, D., Schüssler, M., \& Solanki, S. K. 2004, A\&A, 426, 1075 Baumann, I., Schmitt, D., \& Schüssler, M. 2006, A\&A, 446, 307

Caligari, P., Moreno-Insertis, F., \& Schüssler, M. 1995, ApJ, 441, 886

Chapman, G. A., Cookson, A. M., \& Dobias, J. J. 1997, ApJ, 482, 541

Chapman, G. A., Cookson, A. M., Dobias, J. J., \& Walton, S. R. 2001, ApJ, 555, 462

DeVore, C. R., Boris, J. P., \& Sheeley, N. R. 1984, Sol. Phys., 92, 1

Dikpati, M., de Toma, G., Gilman, P. A., Arge, C. N., \& White, O. R. 2004, ApJ, 601,1136

Fisk, L. A. 2005, ApJ, 626, 563

Gizon, L. 2004, Sol. Phys., 224, 217

Gizon, L., \& Birch, A. C. 2005, Living Rev. Sol. Phys., 2, 6

Gizon, L., \& Duvall, T. L. 2004, in Multi-Wavelength Investigations of Solar Activity, ed. A. V. Stepanov, E. E. Benevolenskaya, \& A. G. Kosovichev (Cambridge University Press), IAU Symp., 223, 41

Hale, G. E., \& Nicholson, S. B. 1925, ApJ, 62, 270

Hoeksema, J. T. 1984, Ph.D. Thesis, Stanford University

Howard, R. F. 1991, Sol. Phys., 136, 251

Kopecký, M., Kuklin, G. V., \& Starkova, I. P. 1985, Bull. Astron. Inst. Czech., 36, 189

Leighton, R. B. 1964, ApJ, 140, 1547

Lockwood, M., Stamper, R., \& Wild, M. N. 1999, Nature, 399, 437

Lockwood, M., Forsyth, R., Balogh, A., \& McComas, D. 2004, Annales Geophys., 22, 1395

Mackay, D. H., \& van Ballegooijen, A. A. 2006, ApJ, 642, 1193

Mackay, D. H., Priest, E. R., \& Lockwood, M. 2002a, Sol. Phys., 207, 291

Mackay, D. H., Priest, E. R., \& Lockwood, M. 2002b, Sol. Phys., 209, 287

Marsch, E., \& Richter, A. K. 1984, J. Geophys. Res., 89, 5386

Schatten, K. H. 1972, in Solar Wind, ed. C. P. Sonett, P. J. Coleman, \& J. M.

Wilcox (Washington, D.C.: NASA SP-308), 44

Schatten, K. H., Wilcox, J. M., \& Ness, N. F. 1969, Sol. Phys., 6, 442

Schrijver, C. J. 2001, ApJ, 547, 475

Schrijver, C. J., \& DeRosa, M. L. 2003, Sol. Phys., 212, 165

Schrijver, C. J., \& Harvey, K. L. 1994, Sol. Phys., 150, 1

Sivaraman, K. R., Gupta, S. S., \& Howard, R. F. 1999, Sol. Phys., 189, 69

Smith, E. J., \& Balogh, A. 1995, Geophys. Res. Lett., 22, 3317

Smith, E. J., \& Balogh, A. 2003, in Solar Wind Ten, ed. M. Velli, R. Bruno, \& F. Malara (American Institute of Physics), AIP Conf. Proc., 679, 67

Smith, E. J., Marsden, R. G., Balogh, A., et al. 2003, Science, 302, 1165

Snodgrass, H. B. 1983, ApJ, 270, 288

Solanki, S. K., Schüssler, M., \& Fligge, M. 2000, Nature, 408, 445

Solanki, S. K., Schüssler, M., \& Fligge, M. 2002, A\&A, 383, 706

Ulrich, R. K. 1992, in Cool Stars, Stellar Systems, and the Sun, ed. J. Bookbinder, \& M. Giampapa (San Francisco, California: ASP), ASP Conf. Ser., 26, 265

Ulrich, R. K., Evans, S., Boyden, J. E., \& Webster, L. 2002, ApJS, 139, 259 van Ballegooijen, A. A., Cartledge, N. P., \& Priest, E. R. 1998, ApJ, 501, 866 Wang, Y.-M. 1993, J. Geophys. Res., 98, 3529

Wang, Y.-M., \& Sheeley, N. R. 1989, Sol. Phys., 124, 81

Wang, Y.-M., \& Sheeley, N. R. 1992, ApJ, 392, 310

Wang, Y.-M., \& Sheeley, N. R. 1995, ApJ, 447, L143

Wang, Y.-M., \& Sheeley, N. R. 2002, J. Geophys. Res., 107, 10

Wang, Y.-M., \& Sheeley, N. R. 2003a, ApJ, 590, 1111

Wang, Y.-M., \& Sheeley, N. R. 2003b, ApJ, 599, 1404

Wang, Y.-M., Nash, A. G., \& Sheeley, N. R. 1989, Science, 245, 712

Wang, Y.-M., Sheeley, Jr., N. R., \& Lean, J. 2002, ApJ, 580, 1188

Wang, Y.-M., Lean, J. L., \& Sheeley, N. R. 2005, ApJ, 625, 522

Wolfson, R. 1985, ApJ, 288, 769

Zhao, X., \& Hoeksema, J. T. 1994, Sol. Phys., 151, 91

Zhao, X., \& Hoeksema, J. T. 1995, J. Geophys. Res., 100, 19 